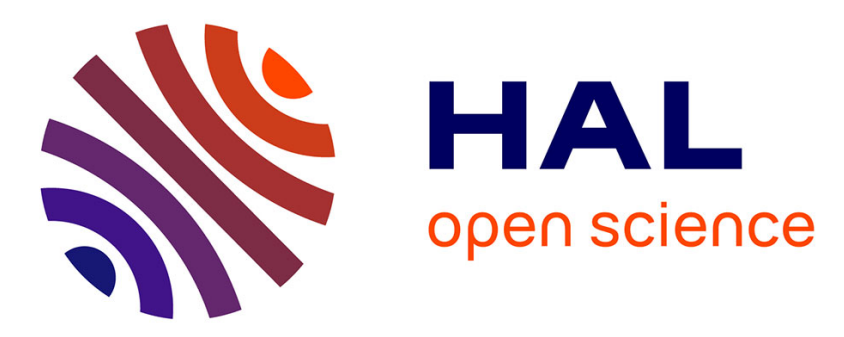

\title{
MAC layer handover mechanism for continuous communication support in healthcare mobile wireless sensor networks
}

João Manuel Leitao Pires Caldeira, Joel José Puga Coelho Rodrigues, Pascal Lorenz

\section{To cite this version:}

João Manuel Leitao Pires Caldeira, Joel José Puga Coelho Rodrigues, Pascal Lorenz. MAC layer handover mechanism for continuous communication support in healthcare mobile wireless sensor networks. Telecommunication Systems, 2015, 60 (1), pp.119-132. 10.1007/s11235-014-9926-z . hal-01318325

\section{HAL Id: hal-01318325 \\ https://hal.science/hal-01318325}

Submitted on 20 Jan 2022

HAL is a multi-disciplinary open access archive for the deposit and dissemination of scientific research documents, whether they are published or not. The documents may come from teaching and research institutions in France or abroad, or from public or private research centers.
L'archive ouverte pluridisciplinaire HAL, est destinée au dépôt et à la diffusion de documents scientifiques de niveau recherche, publiés ou non, émanant des établissements d'enseignement et de recherche français ou étrangers, des laboratoires publics ou privés.

\section{(ㄷ)(1) $\$$}

Distributed under a Creative Commons Attribution - NonCommerciall 4.0 International 


\title{
MAC layer handover mechanism for continuous communication support in healthcare mobile wireless sensor networks
}

\author{
João M. L. P. Caldeira · Joel J. P. C. Rodrigues · \\ Pascal Lorenz
}

\begin{abstract}
The use of wireless sensor networks (WSNs) is growing up in the last few years. Therefore, new challenges arise every day and one of the emerging challenges in WSNs is the nodes mobility support. This feature increases the application areas of these technologies but also raises new challenges to solve. This paper proposes a new handover mechanism, called Hand4MAC (Handover mechanism for MAC layer supporting continuous communication in mobile wireless sensor networks), to deal with body sensors mobility in scenarios where patients are hospitalized. This approach tries to provide continuous monitoring and communication with these sensor nodes when they move across different access points wireless coverage range. The proposed method for medium access control (MAC) layer considers that nodes remain within the same network. The evaluation study of the proposed algorithm was performed by simulation and evaluated in comparison with the well-known RSSI-based handover algorithm. It was concluded that Hand4MAC performs better and reveals promising results for real deployment.
\end{abstract}

J. M. L. P. Caldeira · J. J. P. C. Rodrigues $(\bowtie)$

Instituto de Telecomunicações, University of Beira Interior,

Covilhã, Portugal

e-mail: joeljr@ieee.org

J. M. L. P. Caldeira

e-mail: jcaldeira@it.ubi.pt

J. M. L. P. Caldeira

EST, Polytechnic Institute of Castelo Branco, Castelo Branco, Portugal

J. J. P. C. Rodrigues

University ITMO, Saint Petersburg, Russia

P. Lorenz

IUT, University of Haute Alsace, Colmar, France

e-mail: lorenz@ieee.org
Keywords Body sensor networks - E-health $\cdot$ Mobile health · Mobility · Wireless sensor networks · MAC layer

\section{Introduction}

Wireless sensor networks (WSNs) have grown very fast in the past few years. Their applications in several areas (e.g., military, animal control, environment and biofeedback monitoring) promote the evolution of scientific research in these technologies [1]. However, due to the continuous evolution of these new technologies, several novel challenges and problems arise every day. WSNs are composed by several nodes able to collect sensorial data, deployed along a monitored field. These sensors collect data and send them to remote stations/repositories. This operation is supported by a network infrastructure that allows the transmission of (raw) data from the nodes to the remote entities for storing [2]. Sensor nodes, compliant with wireless technologies, are small devices usually powered by batteries and with low processing capacities [3]. In most of the applications it is not suitable to replace or even to recharge the nodes power sources (batteries). Therefore, if the energy fails in a given node, probably, it will take a long time to get it operating again or forever. This situation could lead to the degradation of the overall network performance. Thus, the lifetime of nodes is a major handicap in WSNs operation. To deal with this weakness, recent studies try to optimize operations in these networks [4]. It is inevitable to compare WSNs with the available and wellknown Wi-Fi networks. Despite their similarities, most of the technologies applied in Wi-Fi are not suitable for WSNs due to their power hungry procedures. Therefore, new challenges were introduced in WSNs due to their limitations in terms of proceeding capabilities and manly on the energy constraints in the nodes [5]. 
The construction of WSNs can vary in their constitution, but usually, a WSN is a combination of several sensor nodes placed along a monitored area (forests, enemy fields, human bodies, etc.) [6,7]. Access points (APs) that interface the access to/from the nodes from/to the remote stations/repositories (usually through the Internet) cover these nodes [8]. Although, in this traditional architecture several aspects are application dependents. One of these aspects is the nodes mobility. Most of the recent WSNs implementation use static nodes. This means that nodes placed in a monitored field remains in their positions all the time and it is not suitable for all the situations. In scenarios where the object/phenomenon/parameter to be monitored changes its position along the time it is important that sensor nodes could move jointly with the monitored parameter. In these scenarios, WSNs became mobility-enable since it is assumed that nodes could move freely around the monitored geographical area [9]. This feature introduces several new challenges and issues on WSNs [10-14]. The network coverage is one of these problems and, consequently, the access by nodes connected to the network infrastructure [15]. If a node moves around a monitored area covered by several APs this means that node must change its AP attachment along the trajectory in order to communicate with the network infrastructure [16]. The process of changing its point of attachment to the network is known as handover. In mobile WSNs scenarios, handover mechanisms support nodes mobility. Recently, several approaches were proposed to optimize the energy wasting with these mechanisms [17-22]. Despite all these proposals sounds promising, the authors consider that solutions are not yet satisfactory. Several possibilities are still unexplored regarding mobility support in WSNs. Then, this paper focuses on handover mechanisms compliant with the standard IEEE 802.15.4 assuming mobility support in controlled scenarios. This handover mechanism was developed concerning the evolution of the handover procedure introduced in [23]. This work was developed regarding a hospital infirmary as a target scenario. The use of WSNs in this scenario supports the monitoring tasks of hospitalized patients [24]. Patients carry a batch of sensor nodes attached to their bodies. Each sensor node collects one or more bio physiological parameters, which are used to evaluate the overall health state of a given patient $[25,26]$. To improve the quality-of-life of hospitalized patients it is important to offer them free walking around the perimeter of an infirmary. These walks should not disturb the monitoring process neither the patients' comfort. The sensors nodes used for patients monitoring are IEEE 802.15 .4 compliant and powered by batteries. The standard IEEE 802.15.4 [27] considers two operation modes, beaconenable and non-beacon. In beacon-enable operating mode it introduces the use of superframe structure to split time into different transmission periods. The standard refers to four time slots, namely, beacon period, contention access period
(CAP), contention free period (CFP), and inactive period. The nodes use the inactive period to enter in sleep mode. This behavior does not guarantee that nodes are reachable all the time since they could lose its contact with the AP during this period without knowing it. In IEEE 802.15.4 non-beacon, it is used the carrier sense multiple access with collision avoidance (CSMA-CA). This transmission mode performs a clear channel assessment (CCA) before sending the radio channel. If the channel is not clear, the algorithm waits for a random time before trying to retransmit. When sending a message, this algorithm could ask for a reception acknowledgement or not (it is configurable) [28]. This IEEE 802.15.4 operation mode could guarantee a continuous communication to nodes in conjunction with a handover mechanism proposed in this paper, named Handover Mechanism for MAC Layer supporting Continuous Communication in Mobile Wireless Sensor Networks (Hand4MAC).

Assuming that nodes belong to the same network, the Hand4MAC algorithm was proposed over IEEE 802.15.4 non-beacon. However, in theory, this approach consumes more energy (due to the inexistence of nodes sleep periods) than a beacon-enable approach. Beacon-enable methodology allows nodes to be unreachable during the sleep periods. Moreover, when nodes are in sleep mode they could not communicate, meaning that APs have to cache the request to nodes during this period of time avoiding real-time communications.

This paper proposes a reliable and robust handover approach over IEEE 802.15.4 non-beacon mode. The proposed method was evaluated by simulation in comparison with the most used handover decision algorithms. The decision to set the best time to perform a handover operation is one of the main issues in these mechanisms. Two main approaches are used, one to establish a new linkafter-break and another to establish a new link-before-break. The Hand4MAC algorithm must guarantee continuous nodes connectivity, so it was used the link-before-break approach.

The rest of this paper is organized as follows. Section 2 reviews the related literature on handover proposals applied to WSNs. Technical details of the proposed handover method are described in Sect. 3 while the network scenario is presented and described in Sect. 4. The performance evaluation of the Hand4MAC mechanism is presented in Sect. 5. Finally, Sect. 6 concludes the paper and point out further research directions.

\section{Related work}

Mobility support is a recent research topic in WSNs. Most of the works proposed up to now in WSNs domain use static elements. Therefore, only few proposals were presented about mobility in WSNs. This paper focuses in handover 
mechanisms for mobility support in WSNs. Thus, this section reviews the most recent and relevant literature in handover mechanisms for mobile WSNs.

In [17], it was proposed a procedure for both inter and intra-mobility support of nodes in controlled WSNs. Since the focus of the contribution proposal is intra-mobility support authors only point out this procedure. The new intramobility support proposed in [17] follows a proxy-based approach. The APs (named proxy agents (PA)) measure the received signal strength indicator (RSSI) value of links. If the value of RSSI with a node falls below a threshold, the PA notifies another PA to start hearing for packets from this node. When a PA receives a packet from the node it informs the old PA by sending it the RSSI value of the received packet. The old PA verifies if the RSSI value is within a valid range and, if it is validated, the old PA informs the new PA that it could register the node. Finally, the old PA notifies the node about the new attachment point with the new PA. Analyzing this procedure it seems that to perform a handover operation a node must still accessible by the old PA to be informed by this one of the new attachment point.

A two phase handover procedure was presented by Fotouhi et al. [18]. This proposal uses two metrics to decide the need for a handover. These two metrics are the velocity of nodes (if available) and the RSSI value of links between nodes and APs. This proposal assumes that nodes send periodic probe messages to its current APs (associated APs) and receive, in return, acknowledge messages. In phase one, if the velocity of the node is unknown, the handover process is triggered when the received RSSI drops below a predefined threshold. Then, with the decision to proceed for a handover in phase one the procedure moves to the phase two. At this phase, the node sends periodic probe requests to all the neighbor APs (multicast) and waits for any probe acknowledge from APs. At this moment, the procedure enters in the decision phase of reassociation with a new AP. If several alternatives are available, the decision is based on a new metric the link quality estimation (LQE). The handover procedure is then finalized by requesting reassociation with the chosen AP.

An approach to support WSN mobility in hospital facilities was proposed in [19]. This approach considers that all nodes have a base network and over the time they can travel to visited networks. When a node moves away from its base network coverage and its link quality drops beyond a predefined threshold it assumes that current router is no longer reachable. After that, the procedure is not clear in what happens, afterwards, for the node. It is not clear if the node starts to find a new router or if it waits until a new router finds it. Although, in this proposal, it seems that nodes allow periods of inaccessibility. When a node enters in a visited network it receives beacon packets from this network coordinator. Next, the node sends an association request with this net- work. After a successful association, negotiated between the base network and the visited network, data sent by the node to the visited network are forwarded to its base network.

All the above-described proposals use the link quality (RSSI) metric between the node and its current point of attachment as the main handover decision, comparing it with a predefined threshold. Continuous messages exchanges are performed for degradation monitoring of this value beyond the predefined threshold. Whether this threshold is crossed, it is time to find a new point of attachment. The Hand4MAC method suppresses the control over link quality value. Therefore, it does not need continuous messages exchanges to get this value.

Petäjäjärvi et al. [20] proposes a soft handover procedure for WSNs. This procedure works as follows: the APs (named gateways $(\mathrm{GW})$ ) periodically spread route advertisement (RA) messages to announce their presence. If a node receives a RA from a GW it checks if it has already register with it or not. If not, the node replies with a registration message. This approach allows nodes to be registered with multiple GWs. Each registered GW is stored into nodes' local memory. Currently, this approach does not perform a real handover operation. Nodes collect all the GWs that are in its range as registered GWs. Then, nodes manage the removal of the GWs that become inaccessible by evaluating the ration of expected RA messages received from each registered $\mathrm{GW}$. Although, the authors of this paper consider this approach very promising and argue the use of multicast RA sent by the GWs (as proposed in ND methods [29]) continues to be a handicap. The proposed approach eliminates the need of this ND feature.

In [30], a mobility approach for WSN based on sensor mobility proxies (SMPs) was proposed. These entities manage the link quality of all mobile nodes in their coverage range. This information is used to decide when a handover should be performed for a specific node. The procedure for handover is executed through a shared backbone that interconnects all the SMP available on the WSN. To keep the information of link quality, SMPs and nodes must exchange periodically probe messages. Next, the proposal of a new handover approach (Hand4MAC) for body sensors with mobility support is presented.

\section{Proposed handover mechanism}

When a sensor node moves across different AP wireless coverage range it should change its registration in order to remain accessible. Knowing the exact moment to change the registration it is one of the most challenging issues in handover procedures. Some of the proposals described in Sect. 2 use the approach register-after-break. This means these solutions allow inaccessibility periods. Thus, using this approach in 
continuous access solutions it is not suitable. It is assumed that nodes must be always reachable. Therefore, the proposed method uses the link-before-break approach.

In the proposal of Hand4MAC method the following assumptions were considered:

(1) The nodes are always within the same network, i.e., its Internet protocol (IP) address never changes.

(2) The nodes must be always reachable (all the time) along the infirmary.

(3) After a short period of time, nodes are well-known by the infrastructure and it is not common changing these nodes, i.e., the nodes remain the same for long periods of time (controlled environment).

Assuming that nodes are always within the same network, it is not necessary to support handover at layer 3 (L3) - open systems interconnection (OSI) model. Therefore, the proposed handover method works over OSI model L2 (MAC layer). Ongoing projects use neighbor discovery (ND) algorithms over L3 to support mobility in WSNs [31]. The current version of "ND optimization for low power and lossy networks (6LoWPAN)" draft (version 18) [29] address some issues related with mobility on WSNs, but it does not deal very well with uninterrupted access to nodes, which is the second assumption.

Figures 1 and 2 present the flowcharts of nodes and APs firmware operations to support the Hand4MAC algorithm. For an easy comprehension of the terminology used in the flowchart representation, it is the same that can be found in the description below.

When a node is new for the network it starts to find an AP for registering. This task is accomplished by sending periodically (at time-to-live-TTL_interval) multicastroute advertisement (RA) messages. If an $\mathrm{AP}$ receives a $\mathrm{RA}$ from a node it creates a new entry in its local cache table (CT) storing the node's address. This table is used by APs to search for all the available nodes there. This search is performed by sending unicast find messages to each node at very short intervals $(\sim 1 \mathrm{~s}$ - an explanation for this value could be found at the end of this section). If a node receives a find message it means that it is within the coverage range of an AP that is not registered there. At this point the node sends an lqiprobe message to the current $\mathrm{AP}$ and, in turn, it receives from this an lqi-probe-acknowledge message. If this probe fails the process suppress the next comparison and proceed directly to the association of this new AP. This probe is used to update the value of the link quality indicator (LQI) for the current AP. Then, the node verifies if the LQI of a new AP is better than the current one. If so it sends a unicast find-acknowledge message to the new AP to perform the new association and sends a unicast break message to the current AP to notify it about the disassociation. After receiving a break message the current AP moves the node entry from the registered table (RT) to the CT. After receiving the find-acknowledge message, the new AP instructs the GW to insert a new entry in access table (AT) with the new register. This new entry is inserted at the top of the table in order to know that newest registrations appear at first in a search for that node. This action avoids that oldest AP also needs to notify the GW about the disassociation. Removing old repeated entries of nodes it is part of the GW AT maintenance tasks.

The registration table (RT) is used to store the information of the registered nodes. This table creates a new entry whenever the AP receives a find-acknowledge message. At this time the node is removed from CT and inserted in RT. It is also associated a timestamp to the registration action for this node. At TTL periods of time expiration the nodes should renew their registration with already registered APs by sending a unicast renew-register message, which returns a renew-register-acknowledge message. If the renewal is not performed the AP moves the node from RT to CT.

When all the nodes become known by all the APs, the handover process is guarantee by the unicast find messages sent from the APs to the unregistered nodes that enter in their coverage range. This method avoids that nodes and APs must exchange regularly messages only to verify that they remain accessible to each other.

\subsection{Assumptions on find messages}

The coverage range of each AP is approximately ten meters, which means that $20 \%$ of this value corresponds to 2 meters. When designing the scenario it was tried to overlap the coverage range of the adjacent APs about $20 \%$ to guarantee continuous access to the nodes. Considering that average speed of a human walking is about 2 meters per second $(\mathrm{m} / \mathrm{s})$ this means he/she takes about $2 \mathrm{~s}$ to travel the previously mentioned $2 \mathrm{~m}$. Using a time interval of $1 \mathrm{~s}$ between find messages in the AP firmware it seems a good choice to guarantee that a node that enters in a non-associated AP's coverage range (overlapped in $20 \%$ with its associated AP's coverage range) receives a find message from this one before losing connection to its associated AP. This way, the handover operation could be performed in a link-before-break manner.

\section{Network scenario}

Monitoring hospitalized people to control their overall health conditions is needed. Depending on diseases, patients need a careful and continuous monitoring control for specific human parameters (e.g., peripheral/core body temperature, pulsation, movement, electroencephalography (EEG), electrocardiography $(\mathrm{ECG}), \ldots$ ). The process of data collection 




Fig. 1 Hand4MAC algorithm—nodes perspective

and turning them available may be performed by a WSN infrastructure. In this paper, the performance of the proposed handover solution is evaluated in an infirmary scenario. The used WSN comprises several body sensor nodes. These nodes are able to collect body parameters and send them wirelessly through APs geographically distributed along the infirmary. These APs are responsible to get network access to all the nodes within their coverage range. The APs distribution assures the coverage of an infirmary. In order to guarantee a continuous communication with nodes, the coverage range of each AP was overlapped by about $20 \%$ with the adjacent ones. Figure 3 depicts an illustration of a hospital infirmary that was used as a scenario for this proposal evaluation. This infirmary comprises nine rooms, a physician 


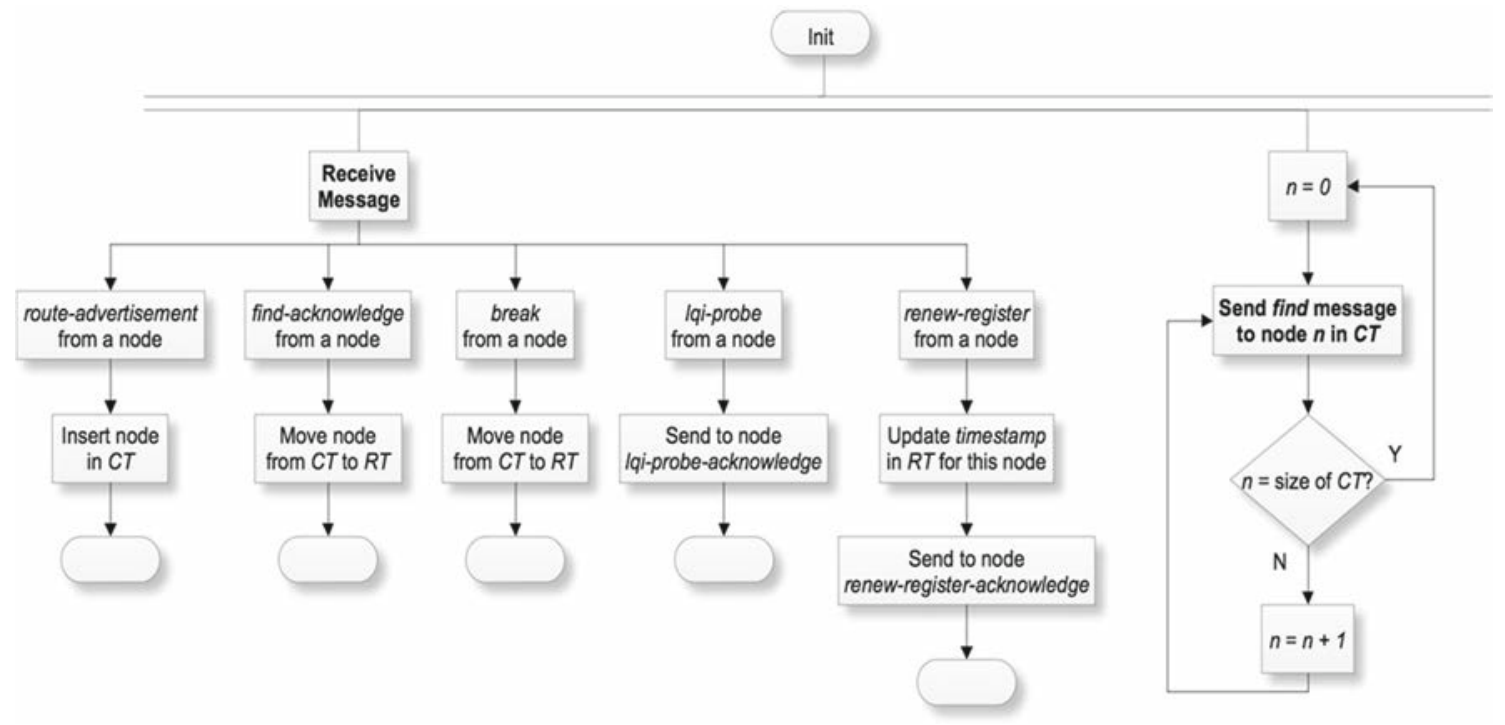

Fig. 2 Hand4MAC algorithm-access point perspective

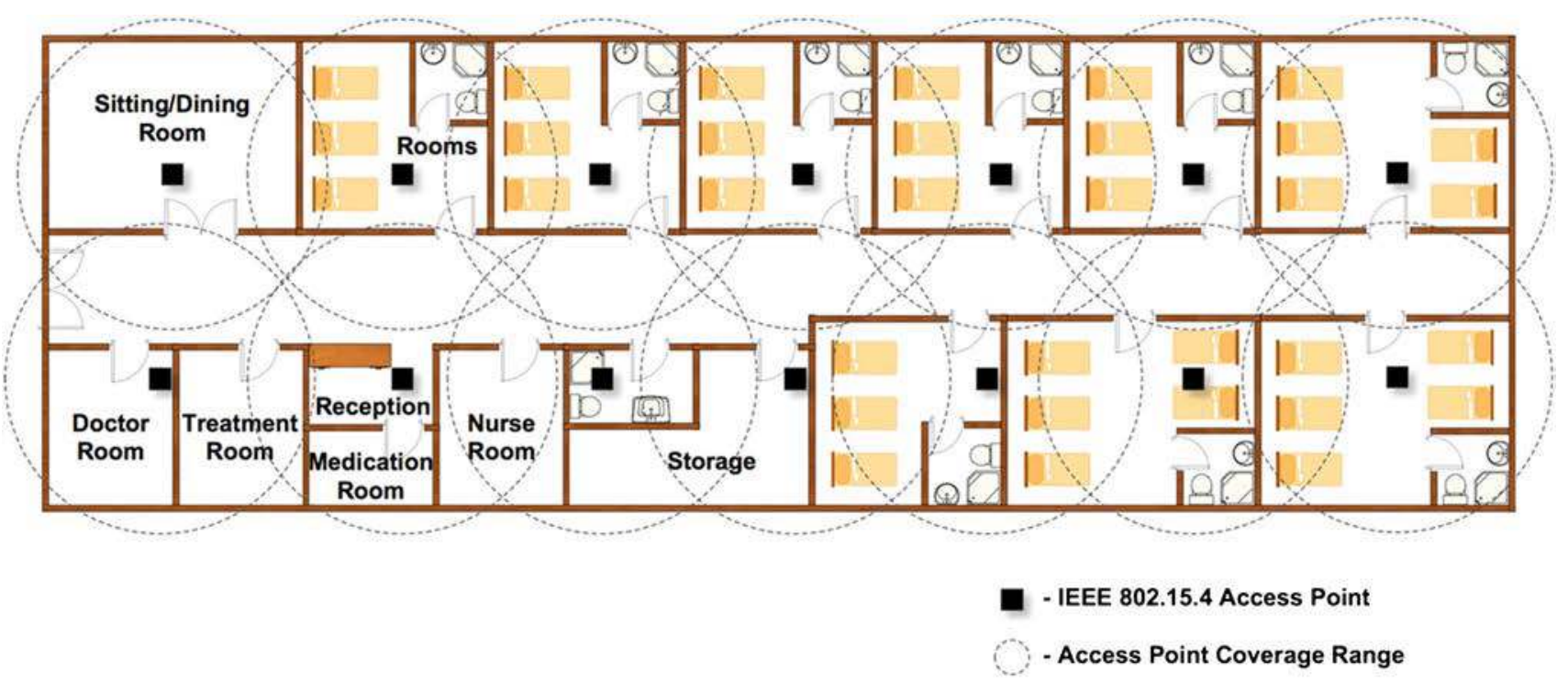

Fig. 3 Illustration of a hospital infirmary covered by access points with IEEE 802.15.4 support

room, a treatment room, a nurse room, a reception, a medication room, a storage area, and a sitting and dining room. To cover all this area fourteen APs with a coverage range of about ten meters were used. As may be seen in Fig. 3, the APs distribution guarantees that coverage ranges of adjacent APs are overlapped about $20 \%$.

Each patient moves freely around the infirmary carrying a batch of small sensor nodes for biofeedback real-time monitoring. These small nodes are powered by batteries and incorporate a CC2420 radio module [32] with IEEE 802.15.4 support [33]. The APs, also compliant with the standard IEEE 802.15 .4 , are powered by electricity. A single gateway (GW) acts as an interface between the APs and the Internet through the hospital network. Figure 4 presents detailed network architecture of the BSN used in the above-described scenario. The GW maintains a table (Access Table) that matches each node to the AP where it is registered. This table is used for routing remote requests to a specific node. In this way, the GW sends this request to the corresponding AP. This table is updated by AP requests whenever a change is performed on its local tables. Each AP uses two local tables (Registration Table and Cache Table) to manage nodes registration on it. In Sect. 3, it was detailed the operation of these tables.

It is assumed that both APs and nodes used for the performance evaluation study of this proposal have about ten meters of coverage range. There are commercial solutions of IEEE 
Fig. 4 Illustration of the sensor network architecture

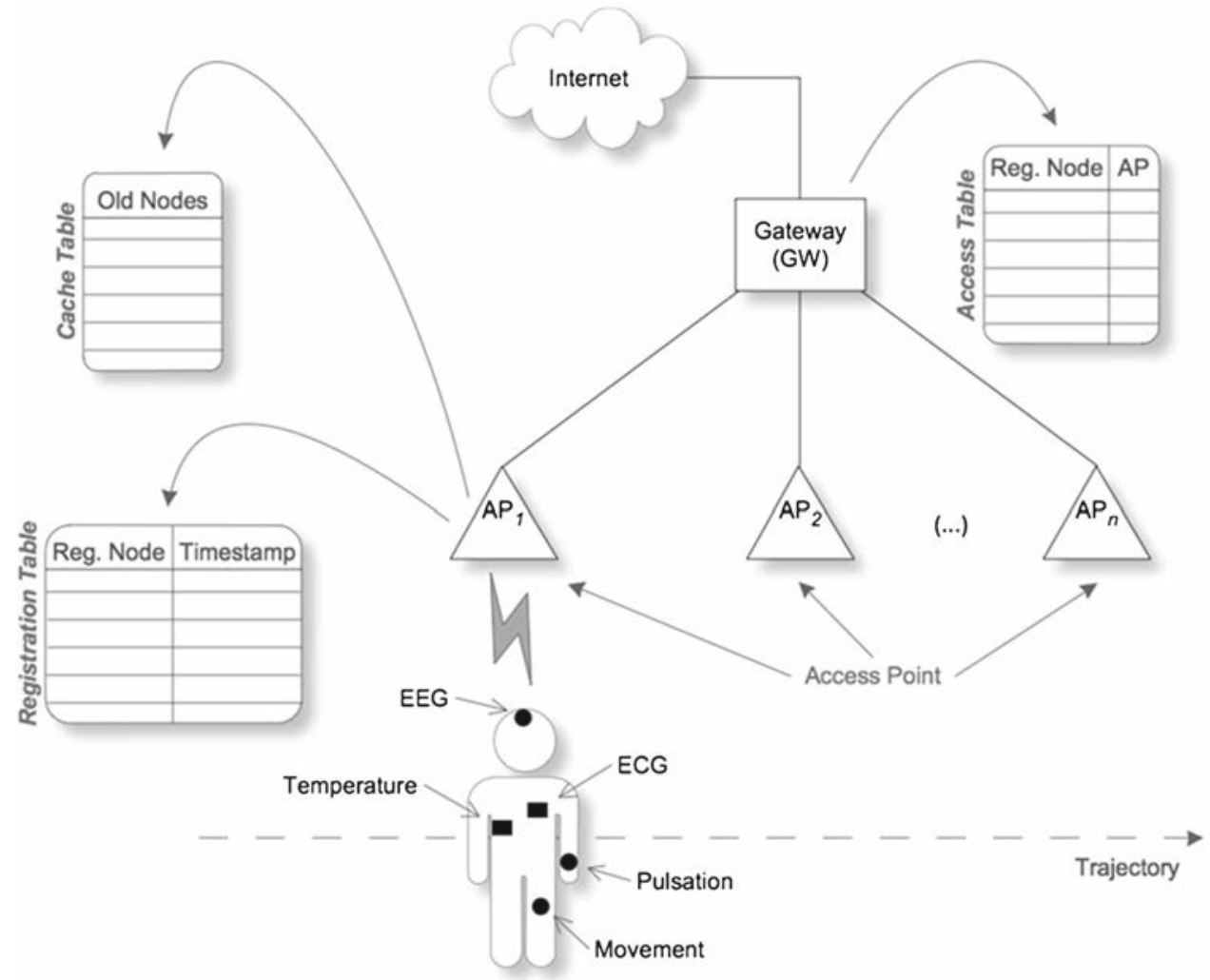

802.15.4 APs with larger coverage ranges, but they are not suitable for this scenario given the need of bidirectional communications. When a patient walks in the infirmary, he/she travels along several coverage ranges by different APs. This situation implies that sensor nodes carried by patients should perform handover between APs for continuous network connectivity.

\section{Performance evaluation}

This section focuses on the performance assessment of the proposed handover mechanism (Hand4MAC) in comparison with RSSI-based handover algorithm [17-19]. This study was performed by simulation using the $\mathrm{OMNeT}++$ [34]. The proposed protocol was developed over IEEE 802.15.4 implementation from MiXiM package [35]. MiXiM is a simulator of wireless and mobile networks for OMNeT++. In this work, the implementation of CSMA-CA transmission mode with non-beacon from the standard IEEE 802.15.4 available in MiXiM package was used. As above described, the proposed protocol was developed at MAC layer. At this layer, a new module to control and process all the messages involved in handover operations was included. The network settings considered in the scenario described at Sect. 4 and the results analysis are presented in the next sub-sections.

\subsection{Network settings}

To evaluate the performance of the Hand4MAC algorithm the network scenario presented in Fig. 3 was used. This scenario was reproduced by the simulation tool, as may be found in Fig. 5. The scenario was created inside an area of 46,0 by 14,5 meters. This area represents the infirmary space. Along this area fourteen static APs were placed. In the simulation scenario, each AP was disposed according to the distribution depicted in the above-described real scenario. These APs run the firmware algorithm described in Sect. 3 (illustrated in Fig. 2). This algorithm was deployed in the MiXiM IEEE 802.15.4 standard implementation.

Six mobile nodes placed in random positions along the scenario area were considered (Fig. 5). In the figure, mobile nodes are represented by a square with a human silhouette. These nodes move randomly with a constant speed of $2 \mathrm{~m} / \mathrm{s}$ to simulate patients walking around the infirmary area. These nodes follow the Hand4MAC algorithm presented in Sect. 3 (Fig. 1). The algorithm was developed for the MiXiM IEEE 802.15.4.

The standard IEEE 802.15.4 implementation used in the study follows the proposal presented in [36] with the same settings. All the nodes are equipped with a $\mathrm{CC} 2420$ radio transceiver [32] and the simulation parameters used for the operation of this module also are presented in [36]. 


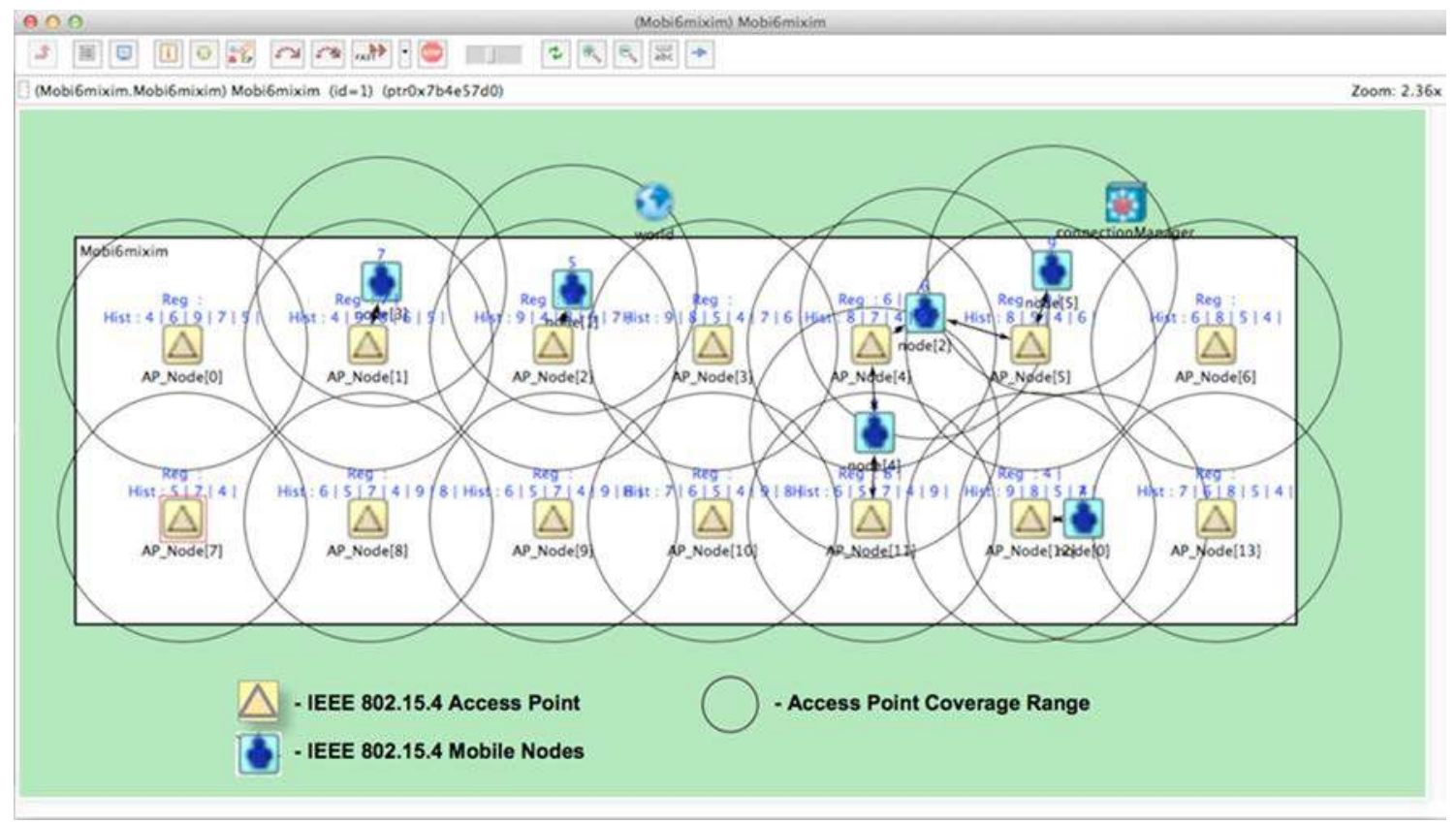

Fig. 5 Simulation scenario regarding a representation of a hospital infirmary area, with the location of access points (squares with a triangle) and the mobile nodes (squares with a human silhouette)

Table 1 Case study 1: network parameters

\begin{tabular}{ll}
\hline Parameter & Value \\
\hline Velocity & $2 \mathrm{~m} / \mathrm{s}$ \\
Time-to-live (TTL) & $5^{\mathrm{a}}$ \\
Simulation time & $1,000^{\mathrm{a}}$
\end{tabular}

${ }^{\text {a }}$ Measured in simulation time units (s)

As above-mentioned, a network performance comparison study between the Hand4MAC algorithm and the handover method used in [17-19] was realized. From now on, to get an easier reference of the method proposed in those works will be referred to as RSSI threshold-based method (RTHM). This study considers two cases for the described scenario.

In the first case study, the simulation parameters were the same for the two methods and are listed on Table 1 . The nodes move randomly with a velocity of $2 \mathrm{~m} / \mathrm{s}$. It was used a time-to-live (TTL) value equal to 5 simulation time units (measured in seconds). This value defines the time intervals among probe messages exchanged between nodes and associated APs. In RTHM, following the algorithm proposed in [18] (phase one), the node receives the probe acknowledge message and compares the RSSI value with the predefined threshold. After this comparison, the algorithm decides if a handover is needed (RSSI $<$ threshold) or not. The simulation time for each experiment was 1,000 simulation time units.

For the second case, the simulation parameters were only changed for the RTHM, as may be seen in Table 2. The veloc-
Table 2 Case study 2: network parameters

\begin{tabular}{lll}
\hline Parameter & Value & \\
\cline { 2 - 3 } & RTHM & Hand4MAC \\
\hline Velocity & $2 \mathrm{~m} / \mathrm{s}$ & $2 \mathrm{~m} / \mathrm{s}$ \\
Time-to-live (TTL) & $1^{\mathrm{a}}$ & $5^{\mathrm{a}}$ \\
Simulation time & $1,000^{\mathrm{a}}$ & $1,000^{\mathrm{a}}$ \\
\hline
\end{tabular}

${ }^{a}$ Measured in simulation time units (s)

ity of the nodes and the movement behavior remains the same as the previous case study. Regarding the TTL value, for the RTHM, 1 time unit was considered. For the Hand4MAC approach, this value remained the same as considered in previous case study (5 time units).

To get representative and meaningful results the batch means method was followed [37]. A set of 30 experiments was performed for each result using different seeds for random calculations. The results under analysis in next subsection represent the average values of 30 experiments.

\subsection{Results analysis}

The performance analysis of the two methods under comparison was performed in two different ways. First, the signaling costs for each method was evaluated in three performance metrics, considering the number of received messages, sent messages, and sent multicast messages. This study was performed for all the 6 mobile nodes considered in the network 
Fig. 6 Signaling overhead of RSSI threshold-based and Hand4MAC mechanisms in function of the number of sent messages, received messages, and sent multicast messages with TTL $=5$ and 6 mobile nodes

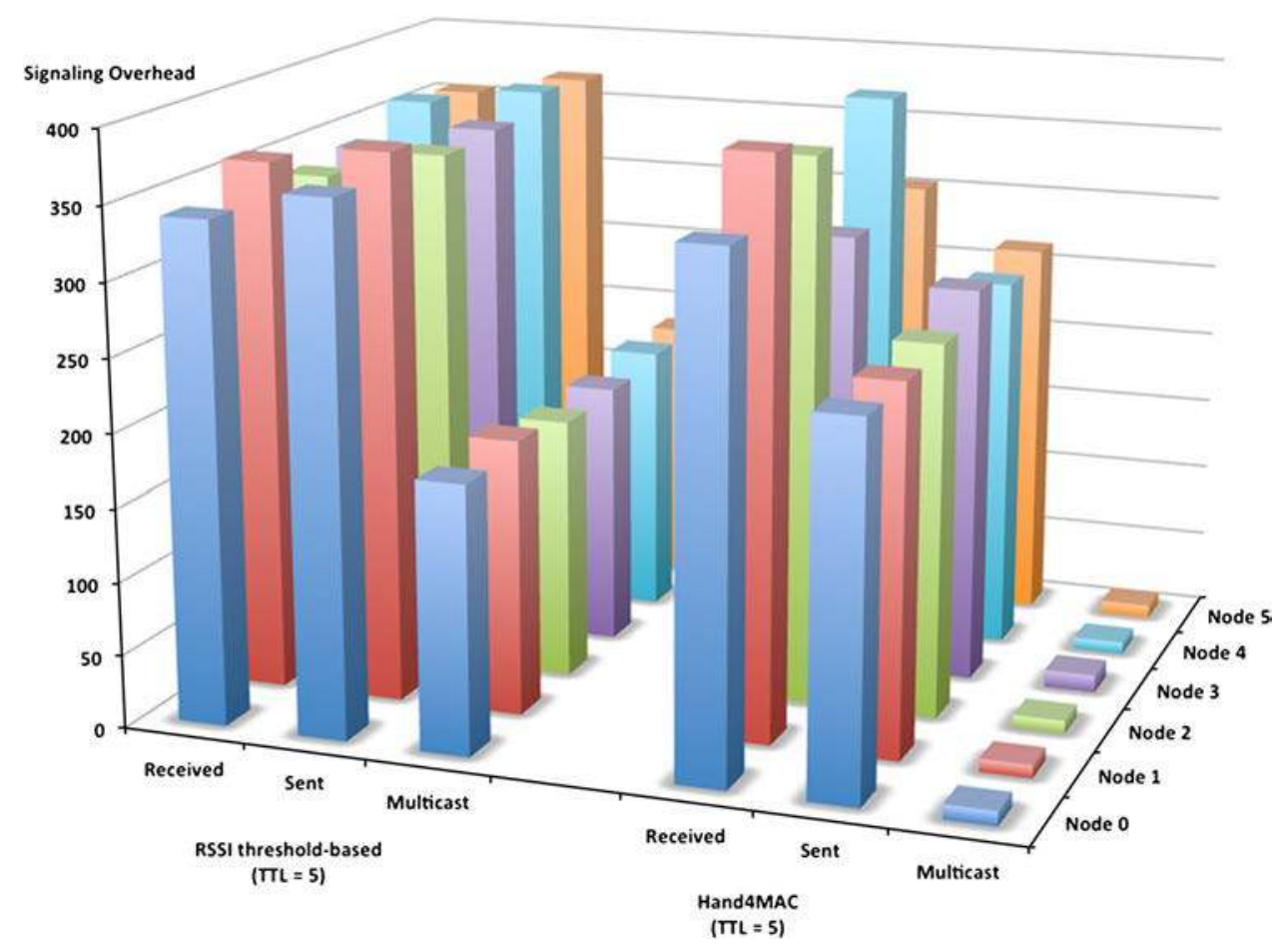

scenario. Then, the connection status between each node and the network backbone was evaluated, i.e., for each node, it was continuously tested if it was remained reachable by the registered AP or not. In other words, if a node could not contact with its registered AP, then it was not connected to the network backbone (so, it was unreachable). The analysis of this metric allows the performance evaluation of the network in terms of the continuous access to nodes.

\subsubsection{Case study 1}

For this first case study, the results of signaling costs obtained for both considered methods are presented in Fig. 6. As may be seen in the figure, it can be concluded that, although the number of received messages were, in average, almost the same for the both mechanisms, the Hand4MAC sent less messages and used significantly less multicast messages. The average number of received messages for RTHM was about 349 and 350 for Hand4MAC. In terms of sent messages, RTHM used an average of 366 messages and 258 for the present proposal. Regarding multicast messages, RTHM sent 184 messages, in average, while Hand4MAC algorithm only sent 10 .

As an example of nodes connection status during experiments, Figs. 7 and 8 depict the connectivity to node 4 using the two handover methods. This result was obtained during the 16th experiment (out of 30). Figure 7 presents the connection status of node 4 using RTHM. Considering that " 1 " means connected to an AP (reachable) and " 0 " not connected (unreachable), it can be seen that using RTHM, the node is unreachable during a longer period of time while Hand4MAC shows better results (Fig. 8). The period of time that each node remains reachable is presented in Table 3 (measured in time percentage). On average, when Hand4MAC algorithm is used, the nodes remained reachable about $98 \%$ of the time while, for RTHM, this value was only about $87 \%$. Analyzing these results it can be concluded that Hand4MAC could almost guarantee a continuous connectivity to nodes unlike the RTHM.

As may be seen in Fig. 8, there are several periods of time where the node is unreachable. It occurs when a node arrives at first time to the coverage range of a given AP and never was registered on it. In upcoming times that node arrives at this AP coverage range it will receive the find message. For RTHM, the unreachable periods of time occurs when a node moves out of a registered AP coverage range. At this time it takes TTL to get a probe message and to compare the RSSI value with the threshold. This means that node only perceives that is out of range just after TTL expires. Then, it starts to look for a new AP.

\subsubsection{Case study 2}

For this second case study, the TTL value used in the RTHM was reduced to 1 time unit. All the other parameters remain the same considered in the case study 1 (Table 2 ). This change aims to reduce the time interval to exchange probe messages between mobile nodes and their registered APs. This means 


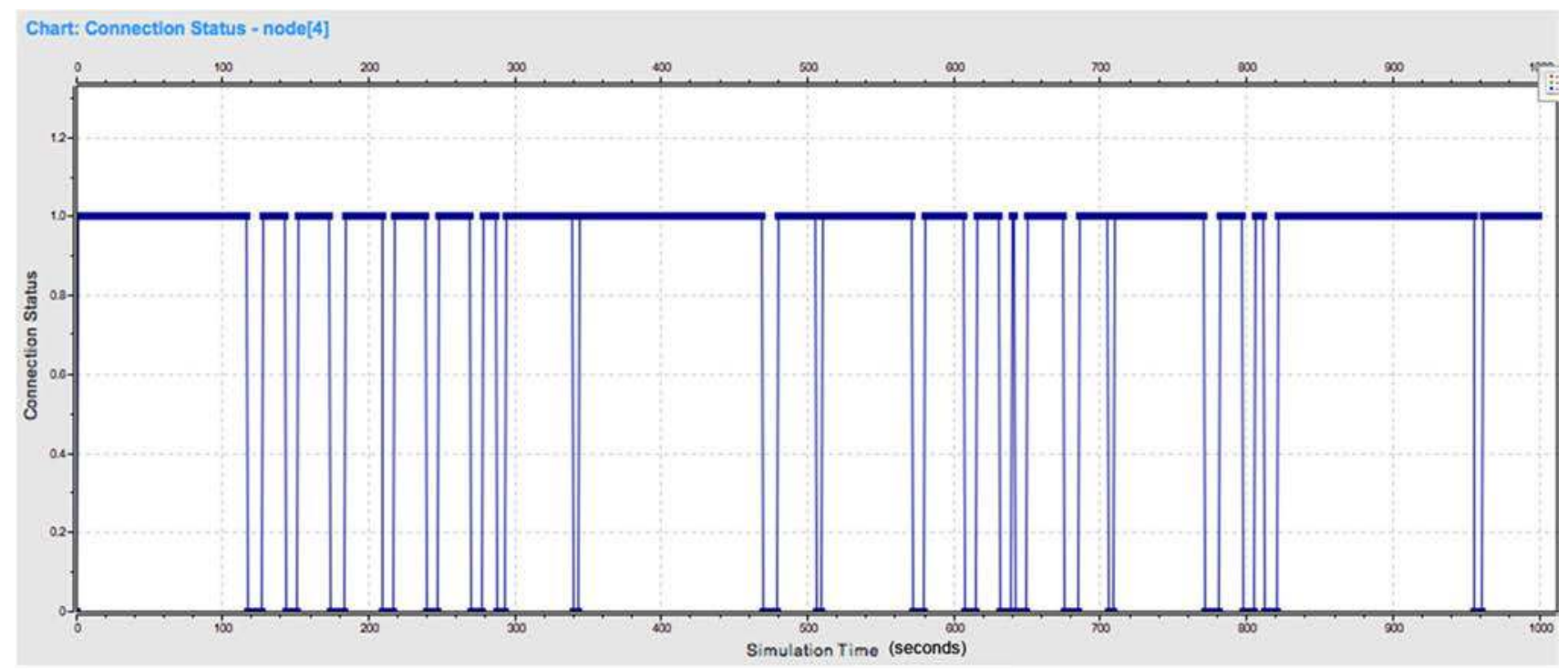

Fig. 7 Connection status of node 4 for RSSI-threshold handover method with TTL $=5$

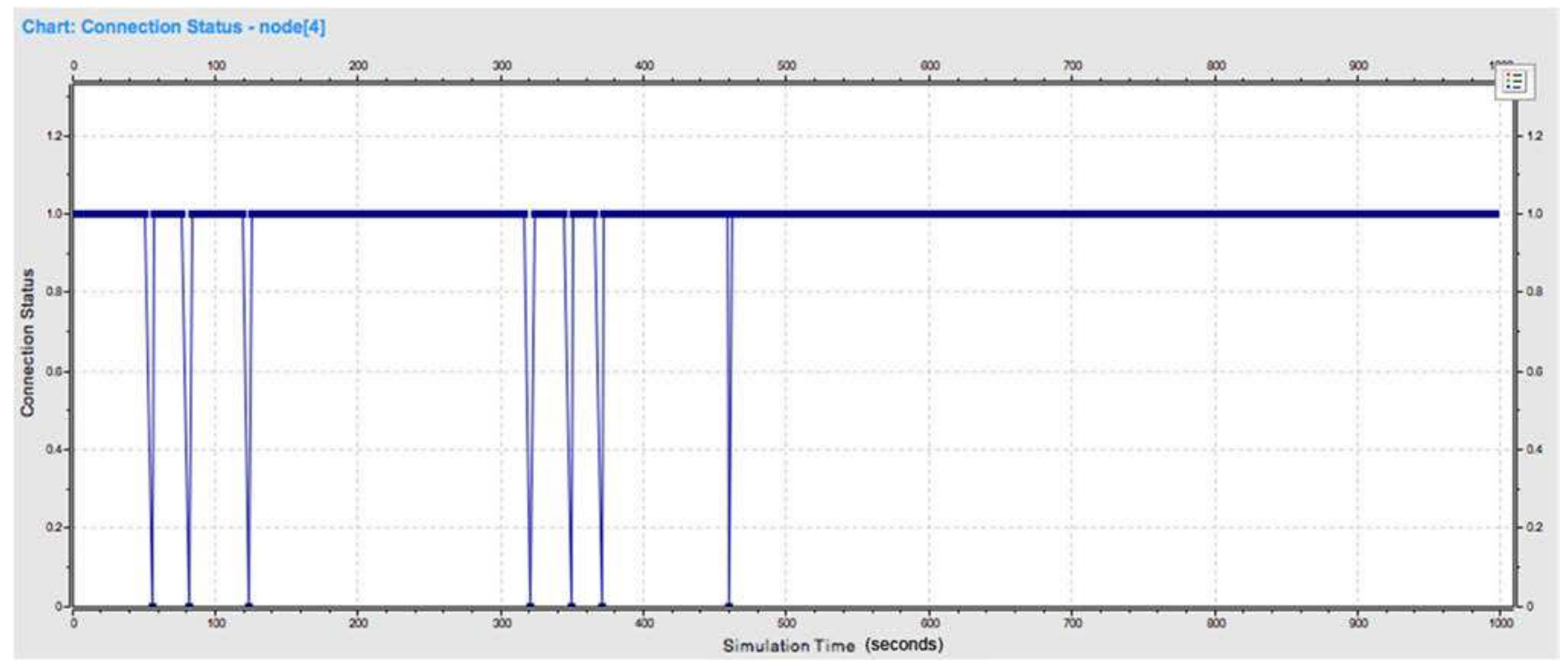

Fig. 8 Connection status of node 4 for Hand4MAC with TTL = 5

Table 3 Percentage of time that each node remains reachable when using the two methods $($ TTL $=5)$

\begin{tabular}{lll}
\hline Node & RTHM $(\%)$ & Hand4MAC (\%) \\
\hline Node 0 & 87.73584 & 97.94988 \\
Node 1 & 88.56839 & 98.12889 \\
Node 2 & 84.08018 & 98.02197 \\
Node 3 & 87.26415 & 97.73195 \\
Node 4 & 88.16037 & 98.49785 \\
Node 5 & 86.51179 & 98.37398 \\
Average & 87.05345 & 98.11742 \\
\hline
\end{tabular}

that nodes perceive earlier they lost contact with their registered APs. As result, it was expected this change increasing the time each node remained reachable by the network.
Furthermore, the connectivity to nodes was increased from about $87 \%$ to almost $98 \%$. This behavior can be observed in Fig. 9 and Table 4. This figure presents the connection status of node 4 during the 16th experiment (out of 30). Although this improvement, the signaling costs increased significantly for all the performance metrics under evaluation, as may be seen in Fig.10. Particularly, the number of received messages was about 1,873 messages, representing an increase of about $536 \%$ when compared to the previous case study. Regarding the number of sent messages, RTHM obtains a result of 1,896 messages on average. This behavior represents about $518 \%$ more messages sent than case study 1 . The number of multicast messages increased also from 184 to 950 messages. This reflects an increase of about $516 \%$. 


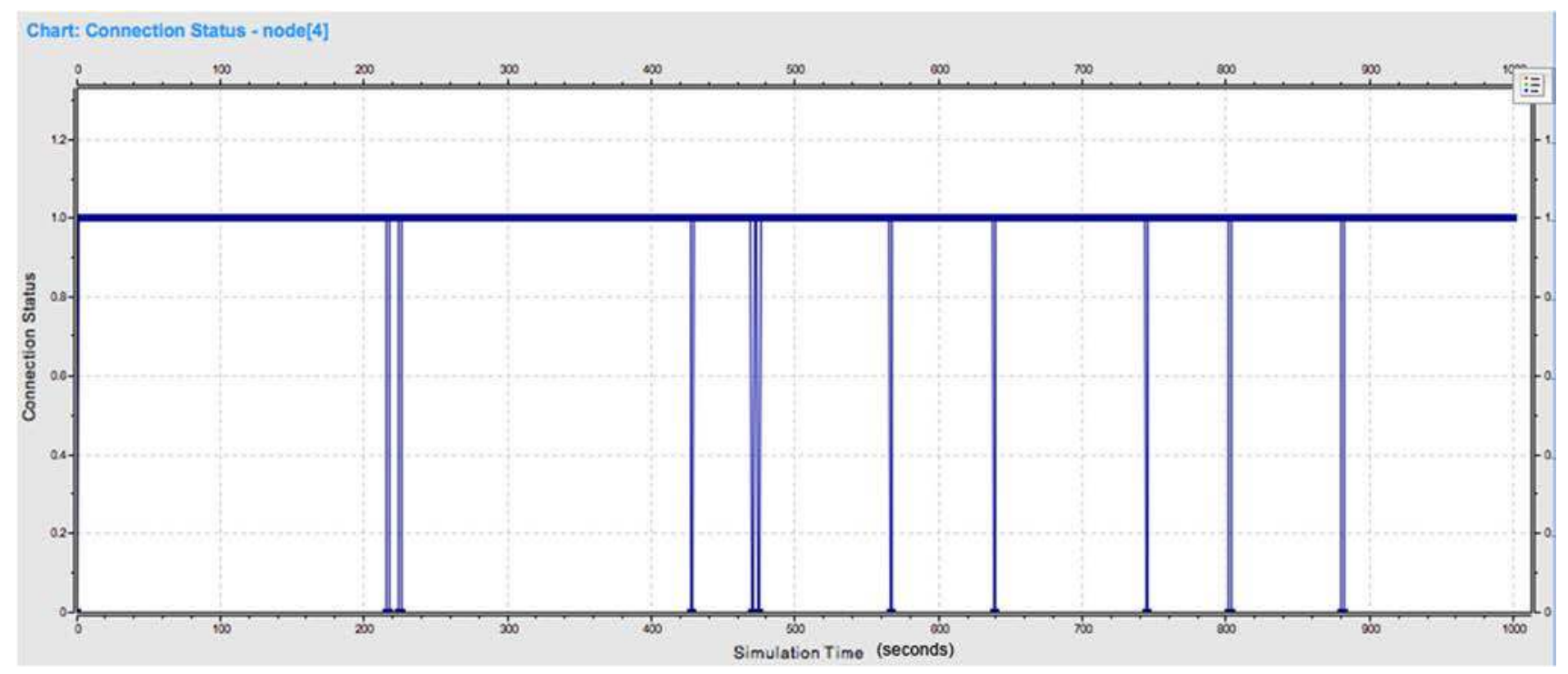

Fig. 9 Connection status of node 4 for RSSI-threshold handover method with TTL = 1

Table 4 Percentage of time that each node remains reachable when using the RTHM with TTL $=1$

\begin{tabular}{ll}
\hline Node & RTHM (\%) \\
\hline Node 0 & 97.68950 \\
Node 1 & 98.70287 \\
Node 2 & 96.10822 \\
Node 3 & 97.89217 \\
Node 4 & 98.50020 \\
Node 5 & 97.51357 \\
Average & 97.73442 \\
\hline
\end{tabular}

Considering the TTL change from 5 to 1 time unit and comparing the Hand4MAC approach with the RTHM, it is observed that connectivity to nodes is now almost the same (about $98 \%$ for both mechanism). In terms of signaling costs all the performance metrics considered in the study increased significantly on RTHM, as may be seen if Fig. 10. The difference among received messages between RTHM (1,873 messages) and Hand4MAC (350 messages) was about $535 \%$. RTHM sent 1,896 messages while Hand4MAC only sent 258 messages. The difference was about $735 \%$ on average. Regarding multicast messages, the difference is about 184 to 10 messages for Hand4MAC showing about $1,840 \%$ more messages for RTHM.

Concluding, for RTHM, when TTL is reduced, the period of time that nodes are reachable increases. However, the number of exchanged messages (sent, received, and sent multicast messages) increases significantly. As a result, the lifetime of nodes batteries will be strongly reduced. Sending and receiving messages represent one of the most energy consumption tasks. Furthermore, the results show that Hand4MAC method can almost guarantee continuous connection to the nodes (about $98 \%$ of the time) with reduced signaling costs. Then, it can be concluded that Hand4MAC mechanism presents better performance than RTHM and can be seen as a promised approach for MAC layer handover for continuous communication support in healthcare mobile WSNs.

\section{Conclusion and future work}

This paper addressed the problem of MAC Layer mobility support in wireless and body sensor networks. This feature provides remote access to nodes moving across several access points' coverage range. In healthcare scenarios like hospital infirmaries it is possible to provide remote access (over a network backbone) to body sensor nodes carried by patients when they move around an infirmary. These nodes collect physiological parameters to monitor their health condition and send this data to remote repositories.

A new handover approach to support continuous communication to nodes in a mobile WSN was proposed. This mechanism, called Handover Mechanism for MAC Layer supporting Continuous Communication in Mobile Wireless Sensor Networks (Hand4MAC), offers overall best performance in comparison with RSSI threshold-based handover method (RTHM). The following performance metrics were considered: number of received messages, sent messages, and sent multicast messages. It was shown that Hand4MAC mechanism is a good solution for continuous connectivity to nodes traveling around an area covered by APs. This method uses a cache table in APs containing all previously registered nodes. Periodically, APs try to contact these nodes. If a node receives a contact from a previously registered AP it decides 
Fig. 10 Signaling overhead of RSSI threshold-based algorithm with TTL $=1$ and Hand4MAC mechanisms with TTL $=5$ in function of the number of sent messages, received messages, and sent multicast messages for 6 mobile nodes

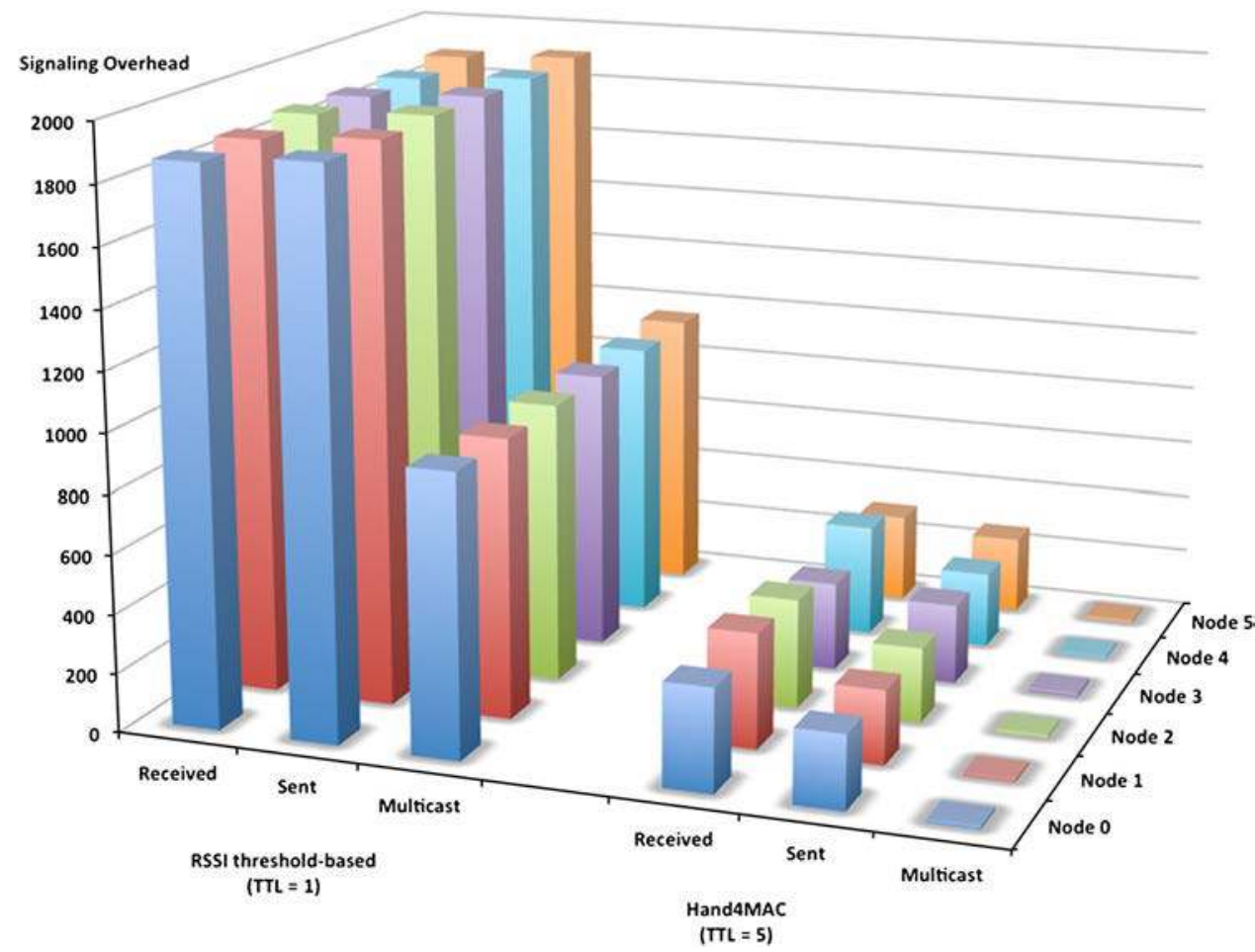

if it is helpful or not to re-register on this AP. This proposal was evaluated by simulation using OMNeT++. The results demonstrate that Hand4MAC method could almost ensure a continuous communication to nodes (about $98 \%$ of the time) with a reduced signaling cost.

Performance evaluation and real deployment of this proposal in a real test-bed should be part of further research works. The optimization of Hand4MAC mechanism also should be considered for future works.

Acknowledgments This work was partially supported by the Instituto de Telcomunicações, Next Generation Networks and Applications Group (NetGNA), Portugal, by Luso-French Program of Integrated University Actions (PAUILF 2010)-Action No. F-CT-10/10, by Government of Russian Federation, Grant 074-U01, by National Funding from the FCT_Fundação para a Ciência e a Tecnologia through the Pest-OE/EEI/LA0008/2013 Project, and by the AAL4ALL (Ambient Assisted Living for All), Project co-financed by COMPETE under FEDER via QREN Programme.

\section{References}

1. Fowler, K. (2009). Sensor survey: Part 1 the current state of sensors and sensor networks. IEEE Instrumentation \& Measurement Magazine, 12(1), 39-44. doi:10.1109/MIM.2009.4762952.

2. Alemdar, H., \& Ersoy, C. (2010). Wireless sensor networks for healthcare: A survey. Computer Networks, Elsevier, 54(15), 26882710. doi:10.1016/j.comnet.2010.05.003.

3. Garcia, J. F. R., Caldeira, J. M. L. P., \& Rodrigues, J. J. P. C. (2010). Wireless body sensor design for intra-vaginal temperature monitoring. In Fifth international conference on body area networks (BodyNets 2010). Corfu Island.
4. Seah, W. K. G., Eu, Z. A., \& Tan, H.-P. (2009). Wireless sensor networks powered by ambient energy harvesting (WSN-HEAP) Survey and challenges. In 1st international conference on wireless communication, vehicular technology, information theory and aerospace \& electronic systems technology (Wireless VITAE 2009), Aalborg (pp. 1-5). doi:10.1109/WIRELESSVITAE.2009. 5172411.

5. Zhou, L., Wang, X., Tu, W., Mutean, G., \& Geller, B. (2010). Distributed scheduling scheme for video streaming over multi-channel multi-radio multi-hop wireless networks. IEEE Journal on Selected Areas in Communications, 28(3), 409-419.

6. Murty, R. N., Mainl, G., Rose, I., Chowdhury, A. R., Gosain, A., Bers, J., \& Welsh, M. (2008). Citysense: An urban-scale wireless sensor network and testbed. In IEEE international conference on technologies for homeland security (2008), Boston (pp. 583-588).

7. Sahin, Y. G. (2007). Animals as mobile biological sensors for forest fire detection. Sensors for Disaster and Emergency Management Decision Making, Sensors, 7(12), 3084-3099.

8. Akyildiz, I. F., Su, W., Sankarasubramaniam, Y., \& Cayirci, E. (2002). Wireless sensor networks: A survey. Computer Networks, Elsevier, 38(4), 393-422. doi:10.1016/S1389-1286(01)00302-4.

9. Francesco, M. D., Das, S. K., \& Anastasi, G. (2011). Data collection in wireless sensor networks with mobile elements: A survey. Transactions on Sensor Networks, ACM, 8(1), 7.1-7.31. doi:10. 1145/1993042.1993049.

10. Ghassemian, M., \& Aghvami, H. (2008). An investigation of the impact of mobility on the protocol performance in wireless sensor networks. In 24th biennial symposium on communications (BSC 2008) (pp. 310-315). doi:10.1109/BSC.2008.4563264.

11. Lambrou, T. P., \& Panayiotou, C. G. (2009). A survey on routing techniques supporting mobility in sensor networks. In 5 th international conference on mobile ad-hoc and sensor networks (MSN 2009), Fujian (pp. 78-85). doi:10.1109/MSN.2009.37.

12. Amundson, I., \& Koutsoukos, X. D. (2009). A Survey on Localization for Mobile Wireless Sensor Networks. In 2nd international 
workshop on mobile entity localization and tracking in GPS-less environments (MELT. (2009), Orlando, FL.

13. Natalizio, E., \& Loscrí, V. (2011). Controlled mobility in mobile sensor networks: advantages, issues and challenges. Telecommunication Systems, Springer, 52(4), 1-8. doi:10.1007/ s11235-011-9561-x.

14. Zhou, L., Chao, H.-C., \& Vasilakos, A. (2011). Joint forensicsscheduling strategy for delay-sensitive multimedia applications over heterogeneous networks. IEEE Journal on Selected Areas in Communications, 29(7), 1358-1367.

15. Mulligan, R., \& Ammari, H. M. (2010). Coverage in wireless sensor networks: A survey. Network Protocols and Algorithms, Macrothink Institute, 2(2), 27-53. doi:10.5296/npa.v2i2.276.

16. Sadiq, A. S., Bakar, K. A., \& Ghafoor, K. Z. (2010). A fuzzy logic approach for reducing handover latency in wireless networks. Network Protocols and Algorithms, Macrothink Institute, 2(4), 61-87.

17. Zinonos, Z., \& Vassiliou, V. (2010). Inter-mobility support in controlled 6LoWPAN networks. In IEEE GLOBECOM Workshops (GC Workshops 2010), Miami, FL (pp. 1718-1723). doi:10.1109/ GLOCOMW.2010.5700235.

18. Fotouhi, H., Alves, M., Koubaa, A., \& Baccou, N. (2010). On a reliable handoff procedure for supporting mobility in wireless sensor networks. In The 9th international workshop on real-time networks (RTN 2010) in conjunction with the 22nd euromicro international conference on real-time systems (ECRTS 2010), Brussels.

19. Jara, A. J., Zamora, M. A., \& Skarmeta, A. F. G. (2010). An initial approach to support mobility in hospital wireless sensor networks based on 6LoWPAN (HWSN6). Journal of Wireless Mobile Networks, Ubiquitous Computing, and Dependable Applications, 1(2/3), 107-122.

20. Petajajarvi, J., \& Karvonen, H. (2011). Soft handover method for mobile wireless sensor networks based on 6LoWPAN. In International conference on distributed computing in sensor systems and workshops (DCOSS 2011), Barcelona (pp. 1-6). doi:10.1109/ DCOSS.2011.5982208.

21. Garcia, M., Sendra, S., Lloret, J., \& Canovas, A. (2011). Saving energy and improving communications using cooperative group-based wireless sensor networks. Telecommunication Systems, Springer. doi:10.1007/s11235-011-9568-3.

22. Zhou, L., \& Chen, H.-H. (2011). On multimedia scheduling with constrained control channels. IEEE Transactions on Multimedia, 13(5), 1040-1051.

23. Caldeira, J. M. L. P., Rodrigues, J. J. P. C., \& Lorenz, P. (2012). Toward ubiquitous mobility solutions for body sensor networks on healthCare. IEEE Communications Magazine, 50(5), 108-115. doi:10.1109/MCOM.2012.6194390.

24. Pantelopoulos, A., \& Bourbakis, N. G. (2010). A survey on wearable sensor-based systems for health monitoring and prognosis. IEEE Transactions on Systems, Man, and Cybernetics, Part C: Applications and Reviews, 40(1), 1-12. doi:10.1109/TSMCC. 2009.2032660

25. Bradai, N., Chaari, L., \& Kamou, L. (2011). A comprehensive overview of wireless body area networks (WBAn). International Journal of E-Health and Medical Communications, IGI Global, 2(3), 1-30. doi:10.4018/jehmc.2011070101.

26. Chaari, L., \& Kamoun, L. (2011). QoS concepts and architecture over wireless body area networks for healthcare applications. International Journal of E-Health and Medical Communications, IGI Global, 2(4), 50-66. doi:10.4018/jehmc.2011100104.

27. IEEE_Std_802.15.4-2006. (2006). IEEE Standard for Information technology-Telecommunications and information exchange between systems-Local and metropolitan area networks-Specific requirements-Part 15.4: Wireless medium access control (MAC) and physical layer (PHY) specifications for low-rate wireless personal area networks (WPANs).

28. Salman, N., Rasool, I., \& Kemp, A. H. (2010). Overview of the IEEE 802.15.4 standards family for Low Rate Wireless Personal Area Networks. In 7th international symposium on wireless communication systems (ISWCS 2010), New York (pp. 701-705). doi:10.1109/ISWCS.2010.5624516.

29. Shelby, Z., Chakrabarti, S., \& Nordmark, E. (2011). Neighbor discovery optimization for low power and lossy networks (6LoWPAN). draft-ietf-6lowpan-nd-18 (work in progress).

30. Silva, R. M., Silva, J. S., Caldeira, J. M. L. P., \& Rodrigues, J. J. P. C. (2011). Mobile multimedia in wireless sensor networks. International Journal of Sensor Networks (IJSNet) Special Issue on Multimedia Data Applications in Wireless Sensor Networks, InderScience Publishers, 11(1), 3-9. doi:10.1504/IJSNET.2012. 045035. (InderScience Publishers).

31. Ko, J., Terzis, A., Dawson-Haggerty, S., Culler, D. E., Hui, J. W., \& Levis, P. (2011). Connecting low-power and lossy networks to the internet. IEEE Communications Magazine, 49(4), 96-101. doi:10. 1109/MCOM.2011.5741163.

32. "Texas Instruments/Chipcon - CC2420". [Online]. (Jan. 2012). Available: http://focus.ti.com/docs/prod/folders/print/cc2420. html.

33. Caldeira, J. M. L. P., Rodrigues, J. J. P. C., Garcia, J. F. R., \& Torre, Idl. (2010). A new wireless biosensor for intra-vaginal temperature monitoring. Sensors, MDPI, 10(11), 10314-10327. doi:10.3390/ s101110314.

34. OMNeT++. [Online]. (Jan. 2012). Available: http://www.omnetpp. org/.

35. MiXiM. [Online]. (Jan. 2012). Available: http://mixim. sourceforge.net/.

36. Rousselot, J., Decotignie, J. D., Aoun, M., van der Stok, P., Oliver, R. S., \& Fohler, G. (2009). Accurate timeliness simulations for real-time wireless sensor networks. In Third UKSim European symposium on computer modeling and simulation, 2009 (EMS 2009), Athens (pp. 476-481). doi:10.1109/EMS.2009.34.

37. Schriber, T. J., \& Andrews, R. W. (1979). Interactive analysis of simulation output by the method of batch means. In Proceedings of 11th conference on winter simulation (WSC 1979) (Vol. 2, pp. 513-526). San Diego, CA: IEEE Press. 